\title{
Umami and salt reduction
}

\author{
Katsuyuki Ando ${ }^{1}$
}

Received: 23 January 2020 / Revised: 27 January 2020 / Accepted: 27 January 2020 / Published online: 19 February 2020

(c) The Japanese Society of Hypertension 2020

An umami substance L-glutamate was found in seaweed broth by the Japanese investigator Kikunae Ikeda in 1908 [1]. The term "umami" has been used worldwide since 1985 when the 1st International Symposium on Umami was held in Hawaii. Umami is one of five basic tastes, including sweetness, sourness, bitterness, and saltiness, and is an independent taste unable to be made via combination of other tastes. A receptor protein for umami [2], as well as for sweetness and bitterness, was detected in taste cells on the tongue and palate epithelium, whereas ion channels for sourness $\left(\mathrm{H}^{+}\right)$and saltiness $\left(\mathrm{Na}^{+}\right)$exist in taste cells. Tasting umami flavors may be a unique sensory function to ameliorate the palatability of foods.

Hayabuchi et al. demonstrated that an umami substance, monosodium L-glutamate (MSG), ameliorated the decrease in palatability caused by salt reduction in students from eight universities and adult attendees of eleven health seminars in Japan $(n=584)$ [3]. The study participants were within a wide range of ages (19 to $>70$ years old), and examinations were performed in Tohoku (the northern area of Japan) to Kyushu (the southern area of Japan). Thus, MSG is a useful substance to help reducing salt intake.

In the present study [3], MSG appeared to elevate palatability at three different salt concentrations in both students and health seminar participants in all areas of Japan where the study was done (Supplementary Figs. 1-B and 2B) [3]. On the other hand, an MSG-induced increase in saltiness was observed only for the $0.3 \%$ salt solution but not for the 0.6 or $0.9 \%$ salt solutions (Supplementary Figs. 1-A and 2-A) [3]. This effect of MSG was lower for the health seminar participants than for the students. Thus, the effect of MSG in ameliorating the suppressed palatability caused by low salt use, which may not be related to its

Katsuyuki Ando

katsua-tky@umin.ac.jp

1 Iguchi Nephrology-Urology-Internal Medicine Clinic Shin-Koiwa, Tokyo, Japan modifying effect on saltiness, might also not be affected by the condition of the human, although some patients with masked hypertension have an impairment of salty taste recognition [4]. In addition, the palatability in the $0.3 \%$ salt plus MSG solution appeared to be slightly higher than that in the 0.6 or $0.9 \%$ salt plus MSG solutions. The taste of umami substance-added food may be more pleasant with low salt levels than with high salt levels.

The palatability of food is determined not only by taste but also by visual, smelling, tactile, and auditory senses as well as by dietary habits and the environment. Thus, someone might consider that the ameliorating action of umami on the palatability of low salt food is tightly related to Japanese dietary habits and the environment. However, umami substances exist in many foods. As a source of umami, Asian populations use shiitake mushrooms, dried kelp and seafood and fermented products with ingredients made from beans, grains, or seafood. On the other hand, European populations utilize umami from tomatoes, cheese, and ham. Thus, umami helps salt reduction worldwide; There are many reports from investigators around the world suggesting that the addition of umami into food allows for salt reduction, as mentioned in the discussion by Hayabuchi et al. [3]. In 2010, the Institute of Medicine of the National Academies (IOM) mentioned in their booklet Strategies to reduce sodium intake in the United States that "It is possible to replace some of the salt in foods with other taste or flavor compounds or through other flavor strategies or techniques. A prominent example of an added compound involves glutamic acid. Some studies have shown that it is possible to maintain food palatability with a lowered overall sodium level in a food when MSG is substituted for some of the salt" [5].

In food processing, salt plays several roles other than that of a seasoning agent, such as conditioning for fermentation, degeneration and dissolution of proteins, and dehydration. In addition, its antiseptic action extends the shelf life of processed food. Potassium chloride $(\mathrm{KCl})$ is one of the major salt substitutes, but its taste is bitter. Reformulated sausages containing MSG combined with amino acids (lysine and taurine) and other umami substances (disodium 
inosinate and disodium guanylate) masked the undesirable sensory attributes associated with the replacement of salt with $\mathrm{KCl}$, allowing for the production of fermented cooked sausages with good sensory acceptance and salt reduction [6]. In addition, the use of a mixture of salts consisting of $\mathrm{NaCl}, \mathrm{KCl}$, and MSG was demonstrated to be a viable alternative for the production of mozzarella, allowing for salt reduction while still maintaining acceptable sensory quality [7]. Thus, MSG may help salt reduction by ameliorating not only the reduced palatability of the product that occurs with salt reduction but also the unpleasant taste of salt substitutes. As mentioned above, the amelioration of reduced palatability with low salt addition by MSG appears to be unrelated to its effect on saltiness. The effect of umami on palatability may be complicated, and our knowledge concerning it is not yet complete.

In addition to MSG, other umami substances (glutamates other than MSG, inosinate and guanylate), herbs, spices, dairy concentrates, lactates, and so on [5], are reported to be possibly useful for compensation of the decreased palatability of a low salt level. Because food is a combination of many nutrients, the combined effect of MSG and other taste compensators on salt reduction should be further examined for the practical use of umami for salt reduction. Undoubtedly, umami is a powerful tool for salt reduction for prevention of the salt-induced progression of cardiovascular disease [8], as suggested by Hayabichi et al. [3].

\section{Compliance with ethical standards}

Conflict of interest The authors declare that they have no conflict of interest.
Publisher's note Springer Nature remains neutral with regard to jurisdictional claims in published maps and institutional affiliations.

\section{References}

1. Ikeda K. New seasonings. Chem Senses. 2002;27:847-9.

2. Nelson G, Chandrashekar J, Hoon M, Feng L, Zhao G, Ryba N, et al. An amino-acid taste receptor. Nature. 2002;416: 199-202.

3. Hayabuchi H, Morita R, Ohta M, Nanri A, Matsumoto H, Fujitani $\mathrm{S}$, et al. Validation of preferred salt concentration in soup based on a randomized blinded experiment in multiple regions in Japaninfluence of umami (L-glutamate) on saltiness and palatability of low-salt solutions. Hypertens Res. 2020. (e-pub ahead of print 29 January 2020; https://doi.org/10.1038/s41440-020-0397-1).

4. Kubota Y, Higashiyama A, Sugiyama D, Nishida Y, Kubo S, Hirata $\mathrm{T}$, et al. Association between impairment of salty taste recognition and masked hypertension based on home blood pressure in Japanese residents: the KOBE study. Hypertens Res. 2018;41:756-62.

5. Committee on Strategies to Reduce Salt Intake, Food and Nutrition Board. Institute of Medicine of the National Academies. Taste and flavor roles of sodium in foods: a unique challenge to reducing sodium intake. In: Strategies to reduce sodium intake in the United States. The National Academies Press, Washington DC.

6. dos Santos BA, Campagnol PC, Morgana MA, Pollonio MAR. Monosodium glutamate, disodium inosinate, disodium guanylate, lysine and taurine improve the sensory quality of fermented cooked sausages with $50 \%$ and $75 \%$ replacement of $\mathrm{NaCl}$ with $\mathrm{KCl}$. Meat Sci. 2014;96:509-13.

7. Rodrigues JF, Gonçalves CS, Pereira JDS, Pinheiro CM. Utilization of temporal dominance of sensations and time intensity methodology for development of low-sodium Mozzarella cheese using a mixture of salts. J Dairy Sci. 2014;97:4733-44.

8. Liu H, Gao X, Zhou L, Wu Y, Li Y, Mai J, et al. Urinary sodium excretion and risk of cardiovascular disease in the Chinese population: a prospective study. Hypertens Res. 2018;41:849-55. 